\title{
Smart grid mechanism for green energy management: a comprehensive review
}

\section{Adila Fakhar, Ahmed M.A. Haidar, M.O. Abdullah \& Narottam Das}

To cite this article: Adila Fakhar, Ahmed M.A. Haidar, M.O. Abdullah \& Narottam Das (2022): Smart grid mechanism for green energy management: a comprehensive review, International Journal of Green Energy, DOI: 10.1080/15435075.2022.2038610

To link to this article: https://doi.org/10.1080/15435075.2022.2038610

\section{Published online: 22 Feb 2022.}

Submit your article to this journal $\sqsubset$

Q View related articles $₫$

View Crossmark data $\asymp$ 


\title{
Smart grid mechanism for green energy management: a comprehensive review
}

\author{
Adila Fakhar ${ }^{a}$, Ahmed M.A. Haidar $\mathbb{D}^{\mathrm{a}, \mathrm{b}}$, M.O. Abdullah $\mathbb{D}^{\mathrm{c}}$, and Narottam Das $\mathbb{D}^{\mathrm{d}, \mathrm{e}}$

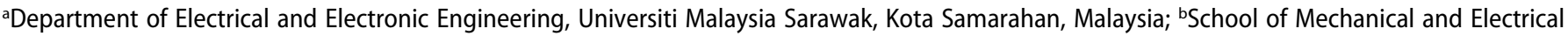 \\ Engineering, University of Southern Queensland, Toowoomba, Australia; 'Department of Chemical Engineering and Energy Sustainability, Universiti \\ Malaysia Sarawak, Kota Samarahan, Malaysia; ${ }^{d}$ School of Engineering and Technology, Central Queensland University, Melbourne, Australia; ${ }^{C}$ Centre \\ for Intelligent Systems, Central Queensland University, Brisbane, Australia
}

\begin{abstract}
The smart grid is not a monolithic system, but rather is a collection of several renewable energy resources and enabling technologies in which, intelligent control is an integral part of its mechanism to improve the utilization of assets. The dynamic characteristics of a smart grid upgrade the conventional system requirements using advanced control strategies to provide continuous power to the load from intermittent renewable generation. The communication networks and control systems that enable the accommodation of distributed generation are crucial technologies in monitoring, protecting, and operating the smart grid in a centralized or decentralized manner. This paper improves the earlier published review articles by exploring the evolution of smart grids in light of renewable energy penetration with associated features. Then, the review gives an overview of notable research works in the literature aimed at developing the management and control of smart energy systems. The reader is provided with an indepth analysis of advanced cloud computing, the internet of things, and blockchain technology with real examples for the related renewable energy projects in smart cities. Furthermore, a special interest has been paid to quantify the performance of communication technologies along with the protocols through the conceptual investigation of real cases using the optimized network engineering tools. The outcomes of the presented review can assist researchers to understand the driving mechanism of smart grid as a route to intelligently utilize renewable energy storage. It is concluded that the amalgamation of blockchain and artificial intelligence for renewable energy management is the key area where the avenue is still open for future research studies.
\end{abstract}

ARTICLE HISTORY

Received 20 December 2020

Accepted 31 January 2022

\section{KEYWORDS}

Renewable resources; green energy management; electric vehicle to grid; demand response; smart grid communication technologies

\section{Introduction}

At present, the electric power system is facing a radical transformation with the rapid use of renewable and sustainable energy resources (RSER) that are growing worldwide. Significant research contributions have been made in the field of power grid integration to quantify the impact of distributed renewable generation on the power grid. This paved the way toward replacing the aging assets and intelligently controlling the natural resources using advanced communication technologies. It is indicated that the current research trends should be undertaken in the area of developing coordination strategies for energy management systems by providing intelligent supervisory functions. With the global movement in the direction of clean energy utilization as an alternative source for the electrification of urban and rural areas, the development of smart grid technologies has become an essential step to make the distribution system self-sustainable and efficiently manageable. The recent advancement in renewable energy technologies and the increased level of power consumption in grid-connected systems may cause power systems to run uneconomically. This is occurring due to the lack of intelligent energy management mechanisms and the aging of power grid components along with the continuous increase in power demand. Indeed, the major factor of blackouts that happened in some countries highlighted the necessity of utilizing intelligent energy management and coordination methods. In this context, the response of control circuits and the availability of information should be improved to enhance the visibility of power grid operation. Other factors, such as the increasing population, decreasing natural reserves, the lifetime of energy storage, and environmental conditions may significantly affect the urban and rural electrifications. To mitigate the above mentioned issues, a new concept so-called "smart renewable energy management system" can overcome the concerns which have been raised about the grid integration with distributed renewable energy resources (Kumar and Bhimasingu 2015; Khan, Haidar, and Othman 2018; Shengrong, Yu, and Liu 2014).

Generally, the smart grid incorporates automation and management systems, intelligent data transfer, power delivery from generation to consumers, and communication systems. The concepts and advancements in smart grid structure are shown in Figure 1 as the flowchart of the system, while the smart grid communication for energy management and automation is depicted in Figure 2 This advanced technology is expected to provide an effective system for energy management in microgrids, which in turn helps in reducing the environmental impact (Rehmani et al. 2018). As the utilization of RSER is growing rapidly worldwide, particularly in developed

CONTACT Ahmed M.A. Haidar ahahmed@unimas.my Department of Electrical and Electronics Engineering, Faculty of Engineering, Universiti Malaysia Sarawak, Kota Samarahan, Sarawak 94300, Malaysia

๑) 2022 Taylor \& Francis Group, LLC 